\title{
Second generation of pepino mosaic virus vectors: improved stability in tomato and a wide range of reporter genes
}

Fabiola Ruiz-Ramón ${ }^{1,2}$, Raquel N. Sempere¹, Eduardo Méndez-López², M. Amelia Sánchez-Pina² and Miguel A. Aranda ${ }^{2 *}$

\begin{abstract}
Background: Vectors based on plant viruses are important tools for functional genomics, cellular biology, plant genome engineering and molecular farming. We previously reported on the construction of PepGFP2a, a viral vector based on pepino mosaic virus (PepMV) which expressed GFP efficiently and stably in plants of its experimental host Nicotiana benthamiana, but not in its natural host tomato. We have prepared a new set of PepMV-based vectors with improved stability that are able to express a wide range of reporter genes, useful for both $\mathrm{N}$. benthamiana and tomato.

Results: We first tested PepGFPm1 and PepGFPm2, two variants of PepGFP2a in which we progressively reduced a duplication of nucleotides encoding the N-terminal region of the coat protein. The new vectors had improved GFP expression levels and stability in N. benthamiana but not in tomato plants. Next, we replaced GFP by DsRed or mCherry in the new vectors PepDsRed and PepmCherry, respectively; while PepmCherry behaved similarly to PepGFPm2, PepDsRed expressed the reporter gene efficiently also in tomato plants. We then used PepGFPm2 and PepDsRed to study the PepMV localization in both N. benthamiana and tomato cells. Using confocal laser scanning microscopy (CLSM), we observed characteristic fluorescent bodies in PepMV-infected cells; these bodies had a cytoplasmic localization and appeared in close proximity to the cell nucleus. Already at 3 days post-agroinoculation there were fluorescent bodies in almost every cell of agroinoculated tissues of both hosts, and always one body per cell. When markers for the endoplasmic reticulum or the Golgi apparatus were co-expressed with PepGFPm2 or PepDsRed, a reorganisation of these organelles was observed, with images suggesting that both are intimately related but not the main constituents of the PepMV bodies. Altogether, this set of data suggested that the PepMV bodies are similar to the potato virus X (PVX) "X-bodies", which have been described as the PVX viral replication complexes (VRCs). To complete the set of PepMV-based vectors, we constructed a vector expressing the BAR herbicide resistance gene, useful for massive susceptibility screenings.
\end{abstract}

Conclusions: We have significantly expanded the PepMV tool box by producing a set of new vectors with improved stability and efficiency in both N. benthamiana and tomato plants. By using two of these vectors, we have described characteristic cellular bodies induced by PepMV infection; these bodies are likely the PepMV VRCs.

Keywords: BAR, DsRed, mCherry, Potexvirus, Viral factory, VRC, Viral replication complex

\footnotetext{
*Correspondence: m.aranda@cebas.csic.es

${ }^{2}$ Centro de Edafología y Biología Aplicada del Segura (CEBAS), Consejo

Superior de Investigaciones Científicas (CSIC), Murcia, Spain

Full list of author information is available at the end of the article
} 


\section{Background}

Vectors based on plant viruses are important tools for functional genomics, cellular biology, plant genome engineering and molecular farming [1-6]. Different strategies have been used to achieve stable and efficient recombinant gene expression from modified plant virus genomes, including gene substitution, gene insertion, modular or deconstructed systems and peptide display fusion [4, 7-9]. These approaches have been applied to some of the most popular plant viruses modified to function as expression vectors, including tobamoviruses, potexviruses, tobraviruses, geminiviruses and comoviruses [10].

We have previously reported on the construction of a set of viral vectors based on pepino mosaic virus (PepMV) [11]. PepMV (species Pepino mosaic virus, genus Potexvirus, family Alphaflexiviridae) has a small single-stranded positive-sense RNA genome of approximately $6.4 \mathrm{~Kb}$ which encodes five open reading frames (ORFs) flanked by $5^{\prime}$ and $3^{\prime}$ untranslated regions (UTRs) [12]. ORF 1 encodes a $164 \mathrm{KDa}$ replicase protein (RdRp) that includes three functional domains, a methyl transferase, a helicase and an RNA polymerase domain [1315], followed by three overlapping ORFs (2-4) which encode the triple gene block (TGB) proteins 1-3 (TGB1 of $26 \mathrm{kDa}$, TGB2 of $14 \mathrm{kDa}$ and TGB3 of $9 \mathrm{kDa}$ ); TGB proteins are involved in cell-to-cell movement, suppression of RNA silencing and architecture of the viral factories [16-19]. ORF 5 encodes the $25 \mathrm{kDa}$ coat protein (CP) required for encapsidation, cell-to-cell movement and suppression of RNA silencing as well [13, 19-21]. In our previous work, up to three different strategies were used to develop the PepMV-based vectors, including substitution of the $C P$ gene and duplication of the $C P$ subgenomic mRNA promoter; in the first case, the vector was unable to move out of the agroinoculated cells, in the second case the insert had poor stability, as it was easily lost during plant to plant passages. The most stable PepMV vector was PepGFP2a, in which the transgene was expressed as a $C P$ fusion through the self-cleaving 2A peptide from foot-and-mouth disease virus (FMDV). PepGFP2a was stable in plant to plant passages in $N$. benthamiana [11], but not in tomato, which is a principal host for PepMV. In the present report, we show results on the construction of a second generation of PepMVbased vectors, for which we wanted to improve their stability and usefulness both in the experimental host $N$. benthamiana and in tomato. We have also used our vectors to gain insights into the distribution of PepMV in $N$. benthamiana and tomato cells. For potato virus $\mathrm{X}$ (PVX), the type member of the genus Potexvirus, infection results in the formation of cytoplasmic "X-bodies" in the periphery of the cell nucleus. X-bodies are inclusion structures induced by the virus that function as viral replication complexes (VRCs). PVX viral proteins TGB1 to 3 play the main roles in VRC architecture, with TGB1 remodelling the actin and host endomembrane system, thereby contributing to the compartmentalization of infection [17] and TGB2/3 becoming inserted into membranous complexes and recruiting TGB1 and CP to the VRC $[16,22]$. In contrast to PVX, the potexvirus alternanthera mosaic virus (AltMV) organizes its VRCs in chloroplasts [23]. For PepMV, Minicka et al. [24] carried out an ultrastructural analysis of tomato tissues infected with different isolates; no clear evidence was produced on the possible nature and localization of PepMV VRCs, even though different cytopathological alterations were observed. Using our PepMV vectors, we have observed cytoplasmic aggregates induced by the virus, normally in the vicinity of the cell nucleus, which may constitute the PepMV VRCs.

\section{Results}

\section{Improvement of PepMV-based vectors for stable GFP expression}

Our previous work showed that the insertion of a GFP transgene in vector PepGFP2a remained stable when infecting $N$. benthamiana, but not tomato plants [11; our unpublished results]. In this vector, the GFP coding sequence was fused in frame to the $C P$ gene through the foot and mouth disease virus (FMDV) sequence encoding the 2A catalytic peptide [11]. Given that the promoter of the CP subgenomic RNA extends downstream of the AUG start codon, the introduction of a sequence duplication in PepGFP2a was necessary in order to keep the $C P$ coding sequence intact [11] (Fig. 1a). To avoid losing the transgene during infection by homologous recombination between duplicated sequences, two new constructs were designed using pBPepGFP2a as the backbone, named pBPepGFPm1 and pBPepGFPm2. For pBPepGFPm1, six synonymous mutations were introduced, one in each of the last six nucleotide triplets of the duplicated sequence, while for $\mathrm{pBPepGFPm} 2$ the last twelve nucleotide triplets were mutated to further reduce sequence duplication (Fig. 1a).

Stability assays were first performed on $N$. benthamiana plants. Leaves were agroinfiltrated with $A$. tumefaciens transformed with pBPepGFP2a, pBPepGFPm1 or pBPepGFPm2 in the presence of the silencing suppressor p19 and GFP expression was monitored under UV light from 3 to $12 \mathrm{dpi}$. All the vectors produced comparable levels of GFP expression in inoculated leaves at $5 \mathrm{dpi}$ (data not shown). At 7 dpi, systemic GFP expression was observed for all the vectors; however, there were larger areas of fluorescent tissues in apical leaves of plants inoculated with PepGFPm2 than with PepGFPm1 or PepGFP2a (Fig. 2a, upper row). This difference increased 


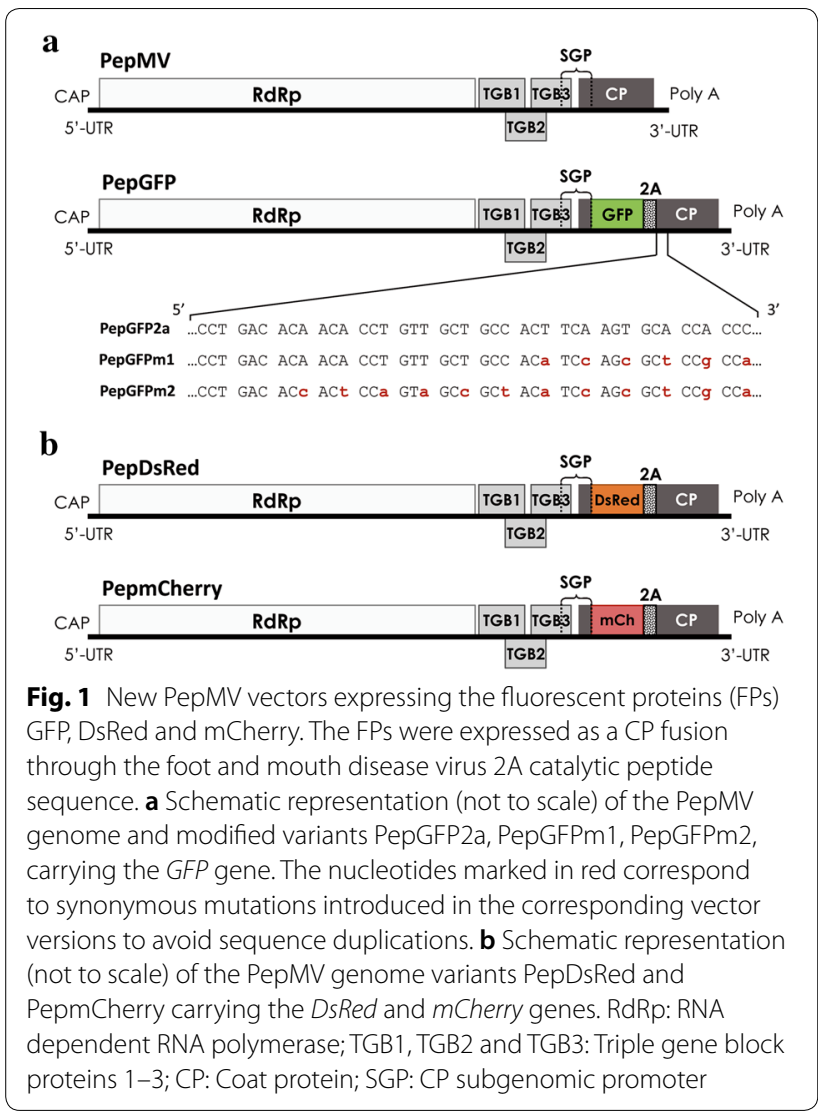

after $12 \mathrm{dpi}$, when plants inoculated with PepGFPm2 produced the greatest amount of GFP fluorescence compared to PepGFPm1 or PepGFP2a (Fig. 2a, lower row). An RT-PCR analysis was carried out using primers to amplify a 1190 bp cDNA fragment for vectors carrying the GFP gene and a 476 bp one for the wild type virus. We sampled inoculated and systemic leaves at $7 \mathrm{dpi}$, and systemic leaves at $12 \mathrm{dpi}$. Results revealed the presence of GFP fusions at 7 and $12 \mathrm{dpi}$ in all samples. Faint wild type $C P$ bands were observed for PepGFP2a at all times post inoculation, and for PepGFPm1 and PepGFPm2 only at $12 \mathrm{dpi}$ (Fig. 2b). The genetic stability of the most promising candidate, PepGFPm2, was tested over infection passages. GFP expression was detected during at least three passages (Fig. 3a) and an RT-PCR analysis of systemic leaves sampled at $10 \mathrm{dpi}$ revealed the presence of the GFP fusion during the three passages (Fig. 3b).

In order to further improve our vector, the insert from pBIN61 was transferred to pJL89, a small and versatile binary plasmid [25], thus obtaining pJL89PepGFPm2. Infection with JLPepGFPm2 resulted in intense GFP fluorescence in agroinfiltrated leaves and evident GFP fluorescence in systemic leaves at $5 \mathrm{dpi}$, whereas PepGFPm 2 produced only abundant although discontinuous GFP fluorescent spots in agroinfiltrated leaves and no

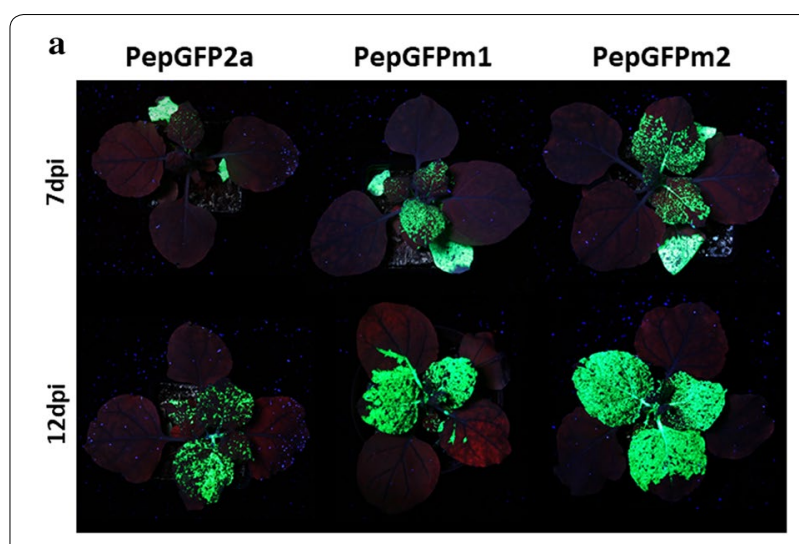

b

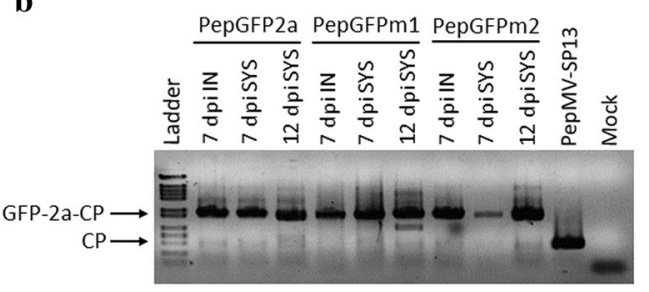

Fig. 2 N. benthamiana plants infected with PepMV vectors expressing GFP. a Fluorescence in N. benthamiana plants inoculated with PepGFP2a, PepGFPm1 or PepGFPm2 under UV light at 7 and $12 \mathrm{~d}$ post inoculation (dpi). $\mathbf{b}$ Agarose gel electrophoresis of RT-PCR products from individual plants to check insert stability in PepMV vectors at 7 and $12 \mathrm{dpi}$. A minimum of 6 plants were used per treatment, with each treatment replicated a minimum of 8 times. All infected plants showed systemic fluorescence at $7 \mathrm{dpi}$. IN: Inoculated leaves; SYS: Systemically infected leaves; PepMV-Sp13, wild type virus

fluorescence in systemic leaves by the same post-inoculation time (Fig. 3c). This suggested that pJL89PepGFPm2 was more efficient than pBPepGFPm2 for agroinoculations, favouring faster accumulation and spread of the tagged virus. In terms of stability through passages, both vectors behaved similarly (Fig. 3b). Next, PepGFPm2 and JLPepGFPm2 were tested in tomato plants. GFP fluorescent spots could be observed only sporadically in agroinoculated leaves and never in systemic leaves (data not shown). An RT-PCR assay to test insert stability showed that at $7 \mathrm{dpi}$, both wild type and fusion inserts could be detected in agroinfiltrated leaves, but at $12 \mathrm{dpi}$ the wild type CP transcript was only detected (see below) indicating that the insert was lost. Thus, these new vectors improved GFP expression levels and stability in $N$. benthamiana but not in tomato plants.

\section{Expression of DsRed and mCherry from modified PepGFPm2 vectors}

We hypothesized that a change of the reporter gene could confer more stability to the transgene in tomato plants. Thus, the GFP gene was replaced with the DsRed 


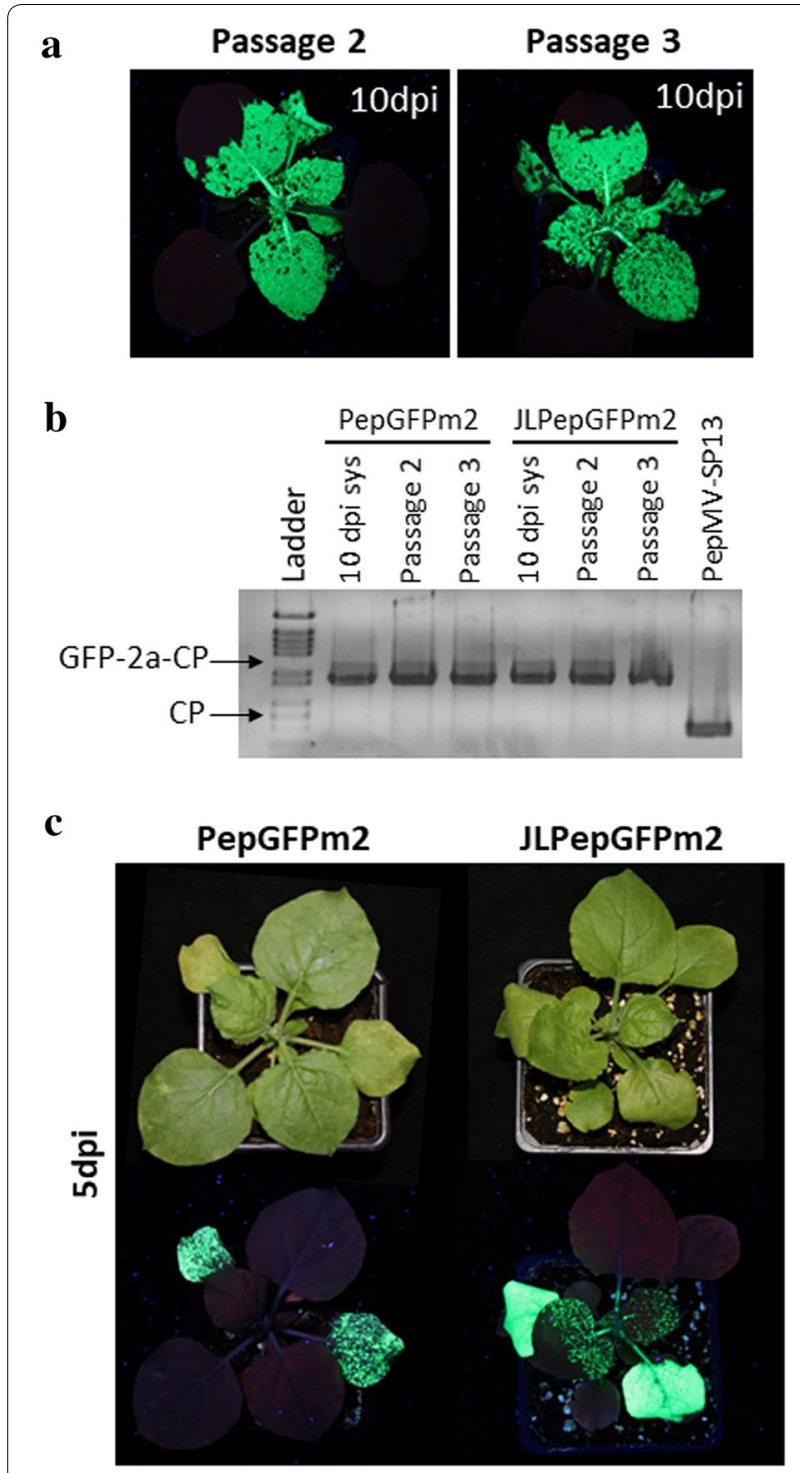

Fig. 3 PepGFPm2 and JLPepGFPm2 vectors and their stability in plant-to-plant passages. a GFP expression from PepGFPm2 at 10 dpi after the second and third passage in N. benthamiana plants. b Agarose gel electrophoresis of RT-PCR products from individual plants to check insert stability during PepGFPm2 passages in $N$. benthamiana plants; samples were taken at $10 \mathrm{~d}$ post inoculation (dpi). c GFP expression in N. benthamiana plants inoculated with PepGFPm2 and JLPepGFPm2 vectors at 5 dpi. A minimum of 6 plants were used per treatment, with each treatment replicated a minimum of 5 times. All infected plants showed systemic fluorescence at $7 \mathrm{dpi}$. SYS: Systemically infected leaves; PepMV-Sp13, wild type virus

or $m$ Cherry genes in the pJL89PepGFPm2 backbone (Fig. 1b). After that, N. benthamiana plants were inoculated with PepDsRed and PepmCherry, and the plants were monitored over 2 weeks for fluorescent protein expression. At $5 \mathrm{dpi}$, it was already possible to observe an intense fluorescence along the veins in systemically

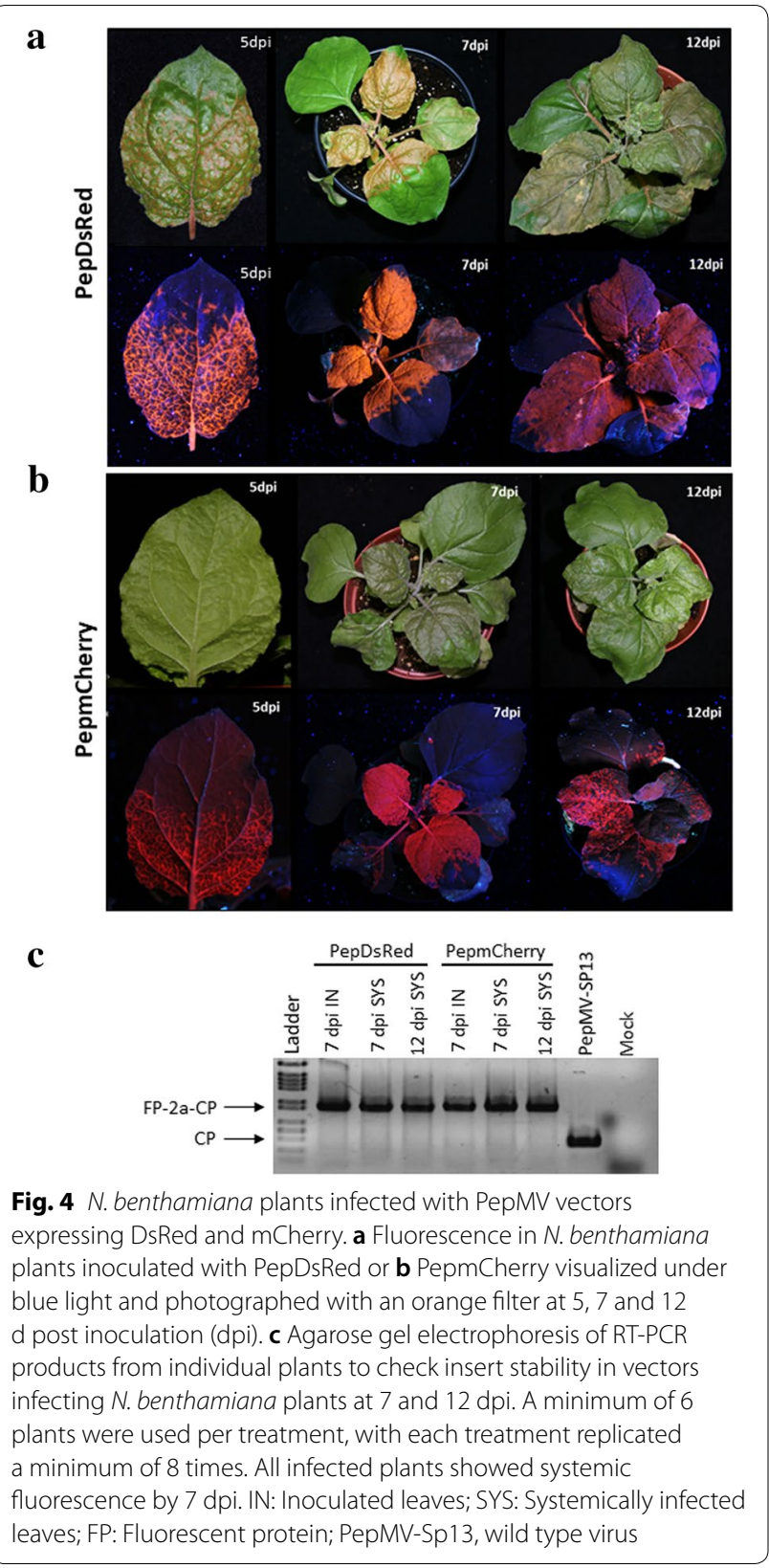

infected leaves, and DsRed or mCherry expression was clearly visible to the naked eye. At $7 \mathrm{dpi}$, intense fluorescence could be observed in systemic leaves for the two vectors (Fig. 4a, b). At 12 dpi, reporter brightness started to decline for PepmCherry while fluorescence was as bright as before for PepGFPm2 and PepDsRed (Fig. 2a, $4 a, b)$. An RT-PCR analysis of infected plant material was performed at different time-points. PepDsRed and PepmCherry should generate 1151 bp and 1184 bp products, respectively, while wild type PepMV should yield a $476 \mathrm{bp}$ product. The presence of DsRed-2a-CP and 
mCherry-2a-CP sequence fusions was detected over time for both constructs and no wild type band was detected (Fig. 4c). An analysis of insert stability during the passages showed that DsRed and mCherry fluorescence was preserved for at least three passages, although mCherry expression became weaker in the third passage (Fig. 5a). Accordingly, an RT-PCR analysis of tissue sampled during passages at $7 \mathrm{dpi}$ showed the product of the DsRed$2 \mathrm{a}-\mathrm{CP}$ sequence fusion in all passages, but a wild type $\mathrm{CP}$ product appeared in the third passage for PepmCherry, indicating that this vector did eventually lose its integrity (Fig. 5b).

After that, tomato plants were inoculated with PepGFPm2, PepmCherry and PepDsRed. We were able to detect DsRed fluorescence in agroinfiltrated and systemically infected leaves but neither GFP nor mCherry expression (Fig. 6a). An RT-PCR analysis confirmed that only the DSRed-2a-CP sequence fusion was stable over time in tomato, while GFP-2a-CP and mCherry$2 \mathrm{a}-\mathrm{CP}$ sequence fusions were detected weakly at 7

$\mathbf{a}$
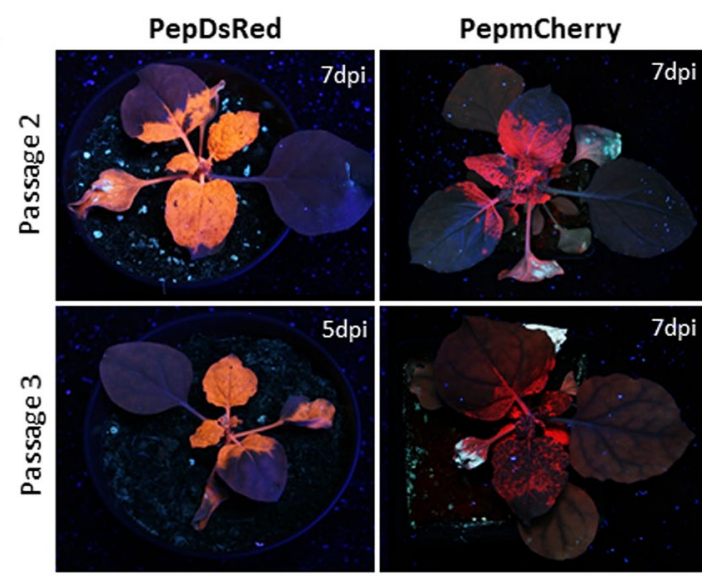

b

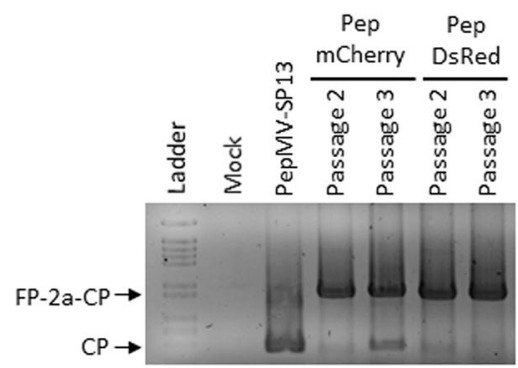

Fig. 5 PepDsRed and PepmCherry stability in plant-to-plant passages. a DsRed and mCherry expression from PepDsRed and PepmCherry after the second and third passages at the times post inoculation (pi) indicated. b Agarose gel electrophoresis of RT-PCR products from individual plants to check insert stability at $7 \mathrm{dpi}$ after passages. A minimum of 6 plants were used per treatment, with each treatment replicated a minimum of 8 times. All infected plants showed systemic fluorescence at $7 \mathrm{dpi}$. FP: Fluorescent protein; PepMV-Sp13, wild type virus dpi, with the signal for the $\mathrm{CP}$ wild type product being much more intense. At 12 dpi PepGFPm2 and PepmCherry had completely lost the insert while the DSRed-2a-CP product was still detectable (Fig. 6b). Passages were performed for PepDsRed, with fluorescence detected for up to two passages (Fig. 6c). An RTPCR analysis showed that the vector was fully stable for the first passage, although from the second passage it was possible to detect the presence of the wild type $\mathrm{CP}$ product to a great extent (Fig. $6 \mathrm{~d}$ ).

In conclusion, the PepDsRed vector yielded robust and stable fluorescence protein expression in both $N$. benthamiana and tomato plants.

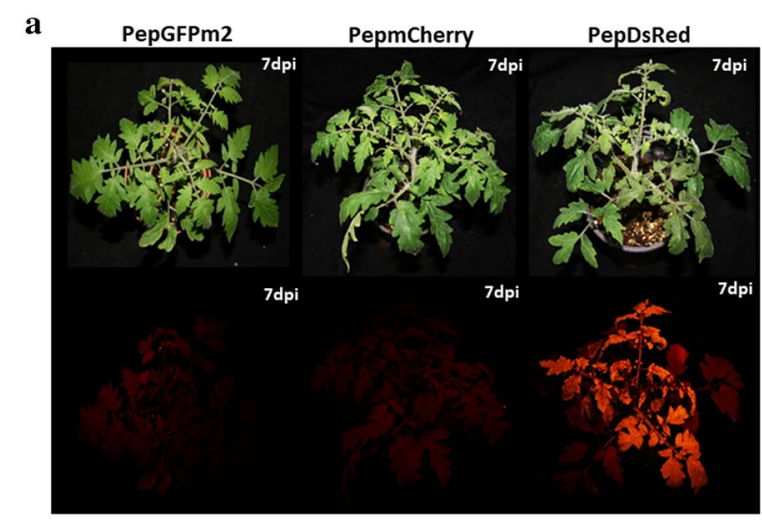

b
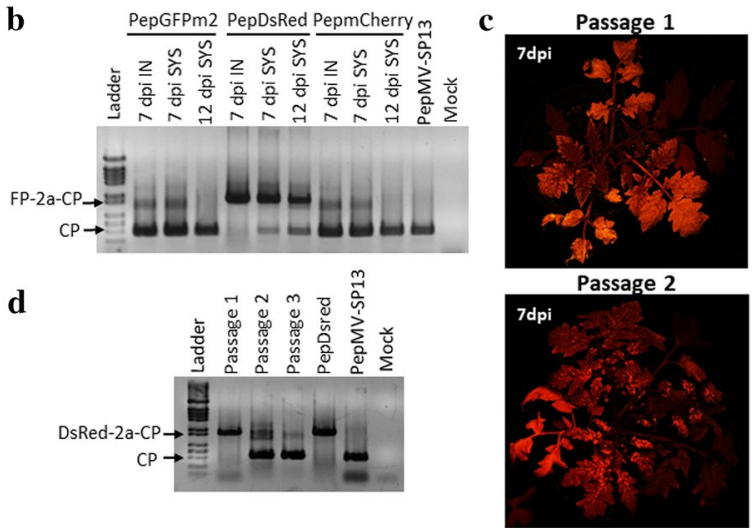

Fig. 6 PepMV vectors expressing fluorescent proteins in tomato plants. a Fluorescence in tomato plants inoculated with PepGFPm2, PepmCherry or PepDsRed at $7 \mathrm{~d}$ post inoculation (dpi). b Agarose gel electrophoresis of RT-PCR products from individual plants to check insert stability in tomato at 7 and $12 \mathrm{dpi}$. c DsRed expression from PepDsRed after the second and third passages at $7 \mathrm{dpi}$. $\mathbf{d}$ Agarose gel electrophoresis of RT-PCR products from individual plants to check insert stability of PepDsRed in tomato passages at $7 \mathrm{dpi}$. A minimum of 6 plants were used per treatment, with each treatment replicated a minimum of 6 times. Only plants infected with PepDsRed showed systemic fluorescence; all of them in passage 1,84\% and none in passages 2 and 3, respectively.IN: Inoculated leaves; SYS: Systemically infected leaves; FP: fluorescent protein 


\section{Using the tagged vectors to analyse PepMV subcellular location}

JLPepGFPm2 and PepDsRed vectors were used to study virus localization in $N$. benthamiana and tomato cells. $N$. benthamiana leaves were agroinfiltrated with each of the two vectors with the same results, while tomato plants were agroinfiltrated only with PepDsRed. Fluorescence emission was analysed under CLSM in agroinfiltrated (Fig. 7) and systemic leaves (data not shown) at 3 and $10 \mathrm{dpi}$, respectively. We observed GFP/DsRed fluorescent bodies in almost every cell in both $N$. benthamiana and tomato samples (Fig. 7a, c). In tomato we counted an average of 85 and 133 fluorescent bodies at 3 and $10 \mathrm{dpi}$, respectively, in an area measuring $755 \mu^{2}$, and always one per cell. In N. benthamiana, there was an average of 62 fluorescent bodies at $3 \mathrm{dpi}$ in the same area, and at $10 \mathrm{dpi}$ we were not able to count them because the tissue was too damaged. These bodies seemed to have a cytoplasmic localization and to be in close proximity to the cell nucleus (Fig. 7b, d). To study their possible relation with cytoplasmic organelles such as the endoplasmic reticulum (ER) and/or the Golgi (G) apparatus, new experiments were conducted in $N$. benthamiana by using ER and G markers [26]. In the absence of PepMV infection, the ER-mCherry marker gave rise to a typical network-like punctuated signal distributed along the cytoplasm (Fig. 7e) [26]. In plants co-agroinfiltrated with ER-mCherry and JLPepGFPm2, an apparent reorganization of the ER was observed (Fig. 7f), with the ER-mCherry's fluorescence mainly localized to aggregates that looked quite similar to the bodies described above for JLPepGFPm2 or PepDsRed infected cells (Fig. $7 \mathrm{f}-\mathrm{h}$ ). Indeed, the ERmCherry fluorescence labelled the same virus-induced bodies as the GFP fluorescence (Fig. 7i, j). However, the signals did not perfectly match (Fig. $7 \mathrm{k}$ ). In a similar analysis using a Golgi-mCherry marker (G-mCherry) [26], an apparent change in the prevalent localization of the G-mCherry signal depending on the presence/ absence of the JLPepGFPm2 infection (Fig. 7l-o) was also observed. Thus, the G-mCherry expressed alone showed its typical punctuated presence spread along the cytoplasm (Fig. 7l) [26] but in cells co-agroinfiltrated with JLPepGFPm2, a significant proportion of the G-mCherry signal localized to the green fluorescent bodies described above (Fig. $7 \mathrm{~m}-\mathrm{o}$ ). The mCherry fluorescence of the G marker (Fig. 7p) appeared around the aggregates labelled in green (Fig. 7q) but the signals did not match when observed with sufficient magnification (Fig. 7r). Thus, both ER and Golgi organelles seemed to be intimately related but not to be main constituents of the bodies formed as the consequence of PepMV infection.

\section{A selectable PepBar vector for high-throughput screenings of tomato}

While PepDsRed seemed to be a sufficiently efficient vector for tomato, a new vector expressing the herbicide resistance $B A R$ gene was considered for completing the set of PepMV-based tools, particularly as a selection tool for massive susceptibility screenings. pBPepBar was constructed with apBPepGFPm2-like backbone (Fig. 1a), with GFP substituted with BAR, the ATG of the $C P$ mutated to AGG and the consensus Kozak sequence added just upstream from the $B A R$ coding sequence (Fig. 8a). This vector was used to inoculate $N$. benthamiana plants, while control plants were inoculated with wild-type virus. At $15 \mathrm{dpi}$, all the plants were treated with a solution containing $0.05 \%$ glufosinate-ammonium (GA). One week after herbicide treatment, plants that were inoculated with the wild-typed virus showed severe wilting symptoms while plants inoculated with PepBar remained healthy (data not shown). After 2 weeks, all the control plants were dead while plants inoculated with PepBar remained healthy and vigorous (Fig. 8b). The presence of the BAR-2A-CP sequence fusion was monitored at several time points with RT-PCR. This analysis revealed that at $14 \mathrm{dpi}$ the vector was stable, as found

\footnotetext{
(See figure on next page.)

Fig. 7 PepMV-induced subcellular bodies in N. benthamiana and tomato plants. a and b CLSM images of JLPepGFPm2 infection in N. benthamiana at $3 \mathrm{~d}$ post inoculation (dpi). $\mathbf{b}$ High magnification of a fluorescent body in JLPepGFPm2 infection to show its cytoplasmic localization and spatial relation with the nucleus. $\mathbf{c}$ and $\mathbf{d}$ CLSM images of PepDsRed infection in tomato at $3 \mathrm{dpi}$. $\mathbf{d}$ High magnification of a fluorescent body in PepDsRed infection. e Distribution of the endoplasmic reticulum marker (ER-mCherry) at $3 \mathrm{dpi}$ in $\mathrm{N}$. benthamiana in the absence of PepMV infection. In the presence of JLPepGFPm2 infection: $\mathbf{f}$ changes of ER-mCherry localization were observed, $\mathbf{g}$ green fluorescent bodies of JLPepGFPm2 and $\mathbf{h}$ merged image of $\mathbf{f}$ and $\mathbf{g}$ to see the matching. High magnification images: $\mathbf{i}$ the distribution of ER-mCherry during the infection, $\mathbf{j}$ the PepMV subcellular body and $\mathbf{k}$ merged image of $\mathbf{i}$ and $\mathbf{j}$ to see the incomplete matching between the red and the green labelling in the body. I Distribution of Golgi-mCherry marker at $3 \mathrm{dpi}$ in N. benthamiana in the absence of PepMV infection. In the presence of JLPepGFPm 2 infection: $\mathbf{m}$ changes of Golgi-mCherry localization were observed (n), green fluorescent bodies of JLPepGFPm2 and $\mathbf{o}$ merged image of $(\mathbf{m})$ and $(\mathbf{n})$ to see the matching. High magnification images of: $\mathbf{p}$ the distribution of Golgi-mCherry during the infection, $\mathbf{q}$ the PepMV subcellular body and $\mathbf{r}$ merged image of (p) and $(\mathbf{q})$ to see the incomplete matching between the red and the green labelling in the body. N, nucleus. Blue colour corresponds to chloroplasts autofluorescence
} 


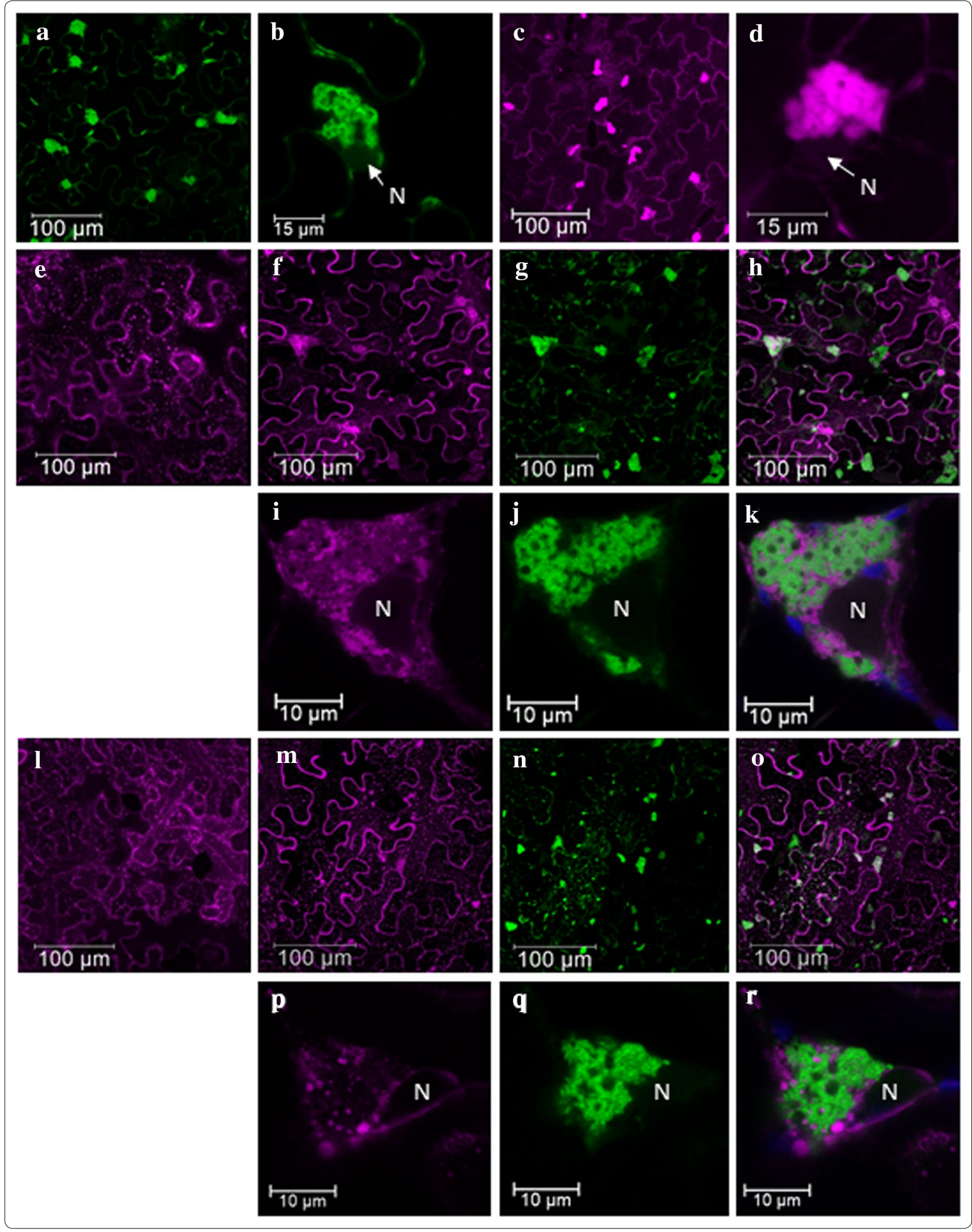


during two successive passages. $N$. benthamiana systemically-infected leaves were used to mechanically inoculate tomato plants, which were treated with $0.05 \%$ GA after $15 \mathrm{dpi}$. Two weeks after the treatment, the controls had completely wilted while the PepBar-infected plants were still alive (Fig. 8c). An RT-PCR analysis indicated that in tomato plants the BAR-2A-CP fusion was stable at 14 dpi. Vector stability was analysed by passaging the virus in tomato, showing that the vector was stable for at least two passages (Fig. 8d).

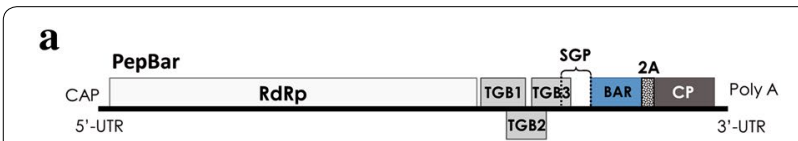

b

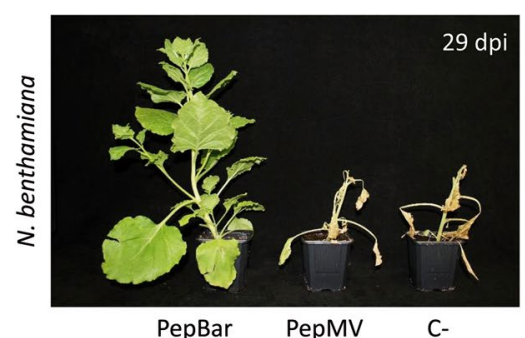

c

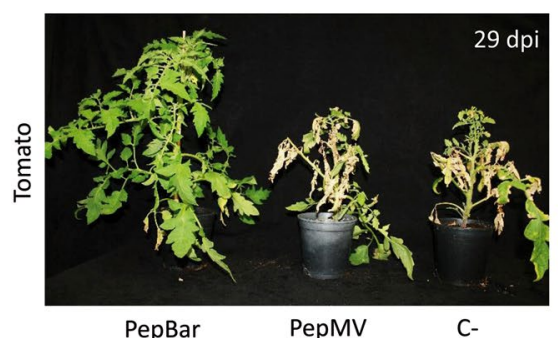

d

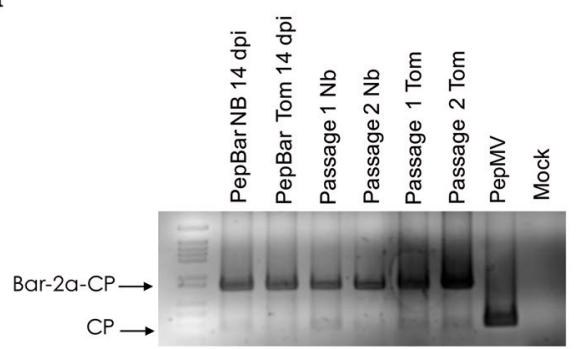

Fig. $8 \mathrm{~N}$. benthamiana and tomato plants infected with the PepMV vector carrying the BAR gene. a Schematic representation of the PepBar genome. $\mathbf{b}$ and $\mathbf{c} N$. benthamiana and tomato plants inoculated with PepBar or PepMV-Sp13 (wild type virus); photo taken 2 weeks after herbicide treatment. $\mathbf{d}$ Agarose gel electrophoresis of RT-PCR products from individual plants to check insert stability in $N$. benthamiana and tomato plants at $14 \mathrm{~d}$ post inoculation (dpi). A minimum of 12 plants were used per treatment, with each treatment replicated a minimum of 3 times. Phenotypes described in the pictures were fully representative of results for the replicates. RdRp: RNA dependent RNA polymerase; TGB1, TGB2 and TGB3: Triple gene block; CP: Coat protein; SGP: CP subgenomic promoter; PepMV wild type virus
In summary, the new PepBar vector seemed to be stable and efficient for selection in both, $N$. benthamiana and tomato plants, thereby becoming a new tool in the PepMV-based toolbox.

\section{Discussion}

A series of PepMV-based vectors had previously been constructed and tested, and this included PepGFP2a, a GFP-expressing vector that was very stable in $N$. benthamiana but unstable in tomato plants [11]. PepGFP2a expresses GFP and $\mathrm{CP}$ as a $\mathrm{N}$-terminal translational fusion linked by the autocatalytic $2 \mathrm{~A}$ peptide, and had a duplication of $36 \mathrm{nt}$ encoding the $\mathrm{N}^{\prime}$-terminal region of the CP [11]. This strategy had previously been used successfully for the creation of a viral vector based on PVX, where the expression of the fusion peptide resulted in the accumulation of significant amounts of the CP fused to the fluorescent protein and lower amounts of the wild type CP necessary for correct encapsidation [27]. Later on, this strategy was used for the creation of vectors based on cowpea mosaic virus (CPMV) [28], bean pod mottle virus (BPMV) [29], wheat streak mosaic virus (WSMV) [30], plantago asiatica mosaic virus (PlAMV) [31] and tobacco mosaic virus (TMV) [32]. An alternative strategy would have been to fuse 2A-GFP to the C-terminus of the $\mathrm{CP}$, but we tried that for our first versions of the PepMV vectors [11] and fluorescence did not spread efficiently in inoculated plants (data not shown). To solve the instability problem of PepGFP2a in tomato, we first worked under the hypothesis that instability could be caused by homologous recombination between repeated sequences during replication, as described in other similar cases [33-37]. Therefore, two new vectors were prepared, PepGFPm1 and PepGFPm2; the length of the duplicated fragment was reduced to $18 \mathrm{nt}$ in PepGFPm1, with no consecutive nucleotide duplication in PepGFPm2. Of the two new vectors, PepGFPm2 was the most stable during passages in $N$. benthamiana plants, in agreement with results described by Draghici and Varrelmann [38] using PVX, where the frequency of transgene loss was conditioned by the length of the homologous sequence. Also, Nagy and Bujarski [39] described that the duplication of just $15 \mathrm{nt}$ could activate homologous recombination in brome mosaic virus (BMV). However, even after the results obtained in $N$. benthamiana, the expression of GFP in tomato was observed only sporadically, perhaps due to the strong negative selection against GFP in tomato, as described for tomato bushy stunt virus (TBSV) [40, 41]. Work done also with TBSV concluded that the expression level of a foreign gene from a viral vector depended on the host into which the vector was inoculated [42]. Thus, two new vectors were designed expressing DsRed or mCherry, which were similar in size to GFP. The two new vectors were 
stable during successive passes in $N$. benthamiana plants, but only PepDsRed was stable in tomato. With PepDsRed an interesting new tool was built, which was useful for both macroscopic and microscopic observations at the cellular or subcellular level in tomato. Next, $N$. benthamiana leaves were inoculated with PepGFPm2 and tomato leaves with PepDsRed to study the subcellular location of PepMV in both hosts. Inoculated tissues were observed using CLSM at 3 and $10 \mathrm{dpi}$. Already at $3 \mathrm{dpi}$, most epidermal cells in both hosts showed PepMV-specific bodies marked with fluorescent proteins, and were found generally adjacent to the nucleus and one per cell. Note that an important proportion of the fluorescent proteins should be free [11], but local aggregation of the fluorescence was significant, implying either that free fluorescent proteins remain within these structures, or that aggregation of the CP fusion protein is such that PepMV-specific bodies are brighter than any other structure within the cell. Structures with similar features to these had previously been observed in infections with other viruses and referred to as viral replication complexes (VRCs), often described as viral factories [43-46]. VRCs could be considered as quasi-organelles responsible for coordinating different processes during viral infection such as replication, protein expression, virion assembly as well as intercellular transport [16]. For PVX, there is extensive knowledge about the VRCs generated during infection; the PVX "X-bodies" $[17,47]$ are built by some of the viral proteins in conjunction with cellular structures or organelles such as the ER or Golgi apparatus [16]. To analyse if there was a relationship between these organelles and the fluorescent bodies detected in cells infected with PepMV, an assay was carried out in which PepGFPm2 was coagroinfiltrated with ER and Golgi apparatus markers, and the distribution of fluorescence was observed by CLSM in agroinfiltrated epidermal cells. Already at $3 \mathrm{dpi}$, it was observed that the ER and Golgi apparatus had been reorganized in the infected cells around the PepMV-induced bodies. This suggests a mechanism of recruitment of these membranous organelles to VRCs, perhaps as a part of a mechanism of protection of the virus factory against the defence mechanisms of the plant, by creating a safe space in which the viral RNA would not be recognized and degraded by the RNA silencing machinery of the plant, and/or as a mechanism to increase the VRC surface, possibly making replication more efficient $[46,48-$ 51]. Further experiments are clearly required, but these observations strongly suggest that the fluorescent bodies detected in both $N$. benthamiana and tomato cells are the PepMV VRCs. Aggregates formed by PepMV particles perhaps related to our observations have been described in infected cells using transmission electron microscopy (TEM) [24].
To complete the toolbox based on PepMV, a vector was built for the expression of the $B A R$ gene for glyphosate resistance; insertion of $B A R$ in the PepMV genome proved to be stable in tomato and provided efficient expression of the transgene and a means of selection. In fact, this vector has been used successfully for carrying out susceptibility tests to PepMV in tomato (data not shown).

\section{Conclusions}

We have significantly expanded the repertoire and improved the stability of viral vectors based on PepMV. This was particularly important for tomato, a crucial host for which not too many efficient vectors were available. We are confident that these vectors will be extremely useful for biotechnological applications [52], but also for the advancement of knowledge on the mechanisms and processes underlying virus/hosts interactions. For instance, by using these vectors, we have described, for the first time, the subcellular bodies that result from PepMV infection, which are likely the PepMV VRCs, opening new venues of research on the potexvirus/hosts molecular interactions.

\section{Methods}

\section{Construction of PepMV-based vectors and mutants}

We used overlapping PCRs [53] for the construction of PepGFPm1 and PepGFPm2. PepGFPm1 and PepGFPm2 fragments were amplified using pBPepGFP2a [11] and PepGFPm1 as templates, respectively. The fragments with the mutations were synthesized using primers CE-Pep303 (5'-CGGAATTGCAGGCACTGGG-3') as forward primer for both constructs and CE-715RC (5'-TGGCGGAGCGCTGGATGTGGCAGCAACAGG TGTTGT-3') and CE-717RC (5'-AGCGGCTACTGGAGTGGTGTCAGGCCCAGGGTTGGA-3') (bold type represents silent mutations and underlined nucleotides the overlapping regions) as reverse primers for PepGFPm1 and PepGFPm2, respectively. The $3^{\prime}$-fragment, covering the $C P$ gene and the $3^{\prime}-\mathrm{UTR}$, was amplified with CE-43 (5'-GGGGTACCGCGGGCCCGGG(T)20$3^{\prime}$ ) as reverse primer for both fragments and the reverse complementary form of CE-715RC and of CE-717RC for PepGFPm1 and PepGFPm2, respectively, as forward primers. The overlapping PCR fragments were cloned into the PTOPO (Thermo Fisher Scientific) vector to generate pTOPOm 1 and pTOPOm2. After that, pTOPOm1 and pTOPOm2 were digested with XmaI and XhoI, the resulting fragments were gel-purified and ligated into pTXLPepXL6 giving rise to pTXLPepGFPm1 and pTXLPepGFPm2. Lastly, pTXLPepGFPm1 and pTXLPepGFPm2 were digested and the resulting fragments carrying PepGFPm1 and PepGFPm2 were subcloned 
into pBIN61 [54] or pJL89 [25], which are binary vectors for expression in plants. For pJL89PepDsRed and pJL89PepmCherry constructs, overlapping PCRs were also used. Three overlapping DNA fragments were amplified in separate PCRs. The first one, a DNA fragment covering the $5^{\prime}$-UTR, the replicase, the TGB genes and the first $36 \mathrm{nt}$ of the $C P$ gene, was amplified using pBPepGFPm2 as template. CE-1955 (5'-CATTTCATTTGGAGAGG GAAAACAAAATAAATAAATAAATATACAAA-3') was used as the forward primer for both constructs, while CE-2049 (5'-GATGACGTTCTCGGAGGAGGCCCT ACTTGAAGTGGCAGCAACAGGTG-3') was used as the reverse primer for pJL89PepDsRed and CE-2089 (5'ACTTGAAGTGGCAGCAACAGGTGTTGTGTC- $3^{\prime}$ ) for pJL89PepmCherry (the underlined primer sequences correspond to the overlapping regions). The DsRed and $m$ Cherry genes were amplified in a second PCR using pEGB 35S:DsRed:Tnos (Addgen plasmid \# 68220) [55] and ER-rk CD3-959 [26] plasmids as templates, respectively. Primers CE-2048 (5'-ACTTCAAGTAGGGCC TCCTCCGAGAACGTCATC-3') and CE-1861 (5'-CTT AAGAAGGTCAAAATTCAGGAACAGGTGGTGGCG $-3^{\prime}$ ) were used to PCR-amplify the DsRed gene, while CE-2090 (5'-ACTTCAAGTAGGGTGAGCAAGGGC GAGG-3') and CE-2091 (5'-TAAGAAGGTCAAAATT CTTGTACAGCTCGTCC-3') were used to PCR-amplify the $m$ Cherry gene (the underlined primer sequences correspond to the overlapping regions). DsRed and $m$ Cherry start codons were removed by changing ATG to AGG (in bold type in primer sequences) to obtain a fusion protein in the same reading frame as the viral protein. The third PCR was performed to PCR-amplify the $2 \mathrm{~A}$ sequence, the $C P$ gene and the $3^{\prime}$-UTR using pBPepGFPm 2 as the template and CE-1954 (5'-ATGCCATGCCGACCC $\left.(\mathrm{T})_{50^{-}} 3^{\prime}\right)$ as the reverse primer for both constructs, and CE-1860 and CE-2092 as the forward primers for pJL89PepDsRed and pJL89PepmCherry, respectively. Fragments resulting from the first and the second PCRs were mixed and amplified in a full-length DNA fragment using CE-1955 as the forward primer for both constructs and CE-1861 as the reverse primer for pJL89PepDsRed and CE-2091 for pJL89PepmCherry. The Gibson Assembly cloning kit (New England Biolabs) was used to join the full-length PCR fragments and the pJL89 vector according to the manufacturer's instructions. For the pBPepBar construct, the $B A R$ gene (coding for phosphinothricin acetyltransferase) was PCR-amplified from plasmid pFGC5941 (GenBank Accession No. AY310901). The PCR was done using primers CE-932 (5'-CGGACCGGTGCCACCATG AGCCCAGAACGACGCCCG-3'), containing an AgeI site (italics) and the consensus Kozak sequence (underlined), and CE-933 (5'-GGAAGCGCTTTTGATCTC GGTGACGGGCAG-3') containing an AfeI site (italics) and the stop codon was removed. Note that the Kozak sequence was inserted just upstream of the $B A R$ coding sequence and downstream of the minimal promoter of the CP subgenomic RNA [11].The resulting product was cloned in the pGEM-T easy (Promega) vector to generate pGEMBar. Then, pGEMBar was digested with AgeI and $A f e \mathrm{I}$ and the resulting fragment carrying the $B A R$ gene was gel-purified and inserted instead of the PDS fragment into a version of pTXLPepPDS2a [11] resulting in pTPepBar; the receptor vector had the CP ATG mutated to AGG and the duplicated $\mathrm{CP}$ subgenomic RNA sequence mutated as in PepGFPm2 (Fig. 1a). Lastly, pTPepBar was digested, and the resulting fragment carrying the PepBar construct was introduced into the AgeI site of the binary vector pBIN61 [54].

\section{Plants, inocula and fluorescence visualization}

Nicotiana benthamiana and tomato cultivar (cv.) M82 plants were grown in a growth chamber set at $25{ }^{\circ} \mathrm{C}$ and $16 \mathrm{~h} / 8 \mathrm{~h}$ (light/dark) conditions. Two weeks-old $N$. benthamiana plants were used for agroinoculations. For this, PepMV constructs were transformed individually into Agrobacterium tumefaciens strain C58C1. Overnight cultures were pelleted through centrifugation at $5000 \times g$ for $8 \mathrm{~min}$ and resuspended in an induction solution $(10 \mathrm{mM}$ MES, pH 5.5, $10 \mathrm{mM} \mathrm{MgSO}$ and $100 \mu \mathrm{M}$ acetosyringone) for $3 \mathrm{~h}$. PepMV cultures were mixed at a 1:3 ratio with $A$. tumefaciens cultures transformed with the $\mathrm{pBp} 19$ vector. Leaves of $N$. benthamiana plants were infiltrated with cultures $\left(\mathrm{OD}_{600}=0.5\right)$ using needle-less syringes. Three weeks-old tomato plants were mechanically inoculated with homogenates of infected $N$. benthamiana systemic leaves in $30 \mathrm{mM}$ sodium phosphate $\mathrm{pH} 8$. Tomato leaves were rubbed with inocula and carborundum. GFP and mCherry fluorescences were monitored daily using a handheld UV lamp (Blak Ray B100-AP lamp, UV products, Upland, CA 91786, USA) while DsRed fluorescence was detected with blue light and an orange filter using a SafeCloner, a device originally designed to visualize EtBr, SYBR Green and SYBR Safe stained gels (Clever Scientific, www.cleaverscientific.com). Pictures were taken with a Canon EOS 400D camera.

\section{Confocal laser scanning microscopy}

Small pieces of agroinfiltrated leaves were mounted onto glass microscope slides and CLSM imaging was performed with a Leica SP8 inverted confocal microscope. The scanning was done by using two objectives, a $40 \times$ magnification oil immersion lens and a $63 \times$ magnification glycerol immersion lens, and the excitation wavelengths used were $488 \mathrm{~nm}$ for GFP and $561 \mathrm{~nm}$ for DsRed and mCherry. Sequential imaging was used for samples co-expressing GFP and mCherry fusion proteins. 
Microscope power settings were adjusted to optimize contrast for each sample. For the CLSM images, we have represented the mCherry fluorescence in magenta.

\section{RNAs preparation and analysis}

Total RNA was isolated using Tri-Reagent (Sigma Chemical Co., St. Louis, MO) and $1 \mu \mathrm{g}$ of total RNA was used for RT-PCR analyses. The RT step was performed with Expand Reverse Transcriptase (Roche) according to the supplier's instructions using CE-43 as the primer. After cDNA synthesis, the region between TGB3 and the $C P$ was amplified using primers CE-2308 (5'-CCATTGTCA GGCCATCATTGAC-3') and CE-2309 (5'-GAACTC TGCACATCAGCATATGC- $3^{\prime}$ ). DNA products were resolved by $1 \%$ agarose gel electrophoresis and stained with ethidium bromide.

\section{Herbicide treatment}

Two weeks after the inoculation with PepBar, infected plants were treated with the herbicide Finale (BAYER CropScience, Victoria, Australia), which contains glufosinate-ammonium (GA) as active ingredient at a final concentration of $0.05 \% \mathrm{GA}(\mathrm{w} / \mathrm{v})$ in deionized water. Plants were photographed $15 \mathrm{~d}$ after the treatment.

\begin{abstract}
Abbreviations
PepMV: pepino mosaic virus; CP: coat protein; dpi: days post-inoculation; RT-PCR: reverse transcription-polymerase chain reaction; CLSM: confocal laser scanning microscopy; ER: endoplasmic reticulum; G: golgi apparatus; GA: glufosinate-ammonium; VRCs: viral replication complexes.
\end{abstract}

\section{Acknowledgements}

We thank Mari Carmen Montesinos (CEBAS-CSIC) for her technical assistance and Mario Fon (mariogfon@gmail.com) for his editorial assistance.

\section{Authors' contributions}

FRR and RNS carried out most of the experimental work and co-participated in the conception of the work. FRR wrote the manuscript. EML and MASP participated in the cell biology experiments. LD supervised the laboratory work for the generation of the DNA constructs. MAA is the principal investigator, conceived the project and supervised the experiments and the writing of the manuscript. All authors read and approved the final manuscript.

\section{Funding}

Work in MAA lab was supported by Grant AGL2015-65838R (Ministry of Economy, Industry and Competitiveness; Spain). FRR and EML were recipients of fellowships 18989/FPI/13 (Fundación Séneca de la Región de Murcia, Spain) and BES-2013-064540 (Ministry of Economy, Industry and Competitiveness; Spain), respectively. We acknowledge support of the publication fee by the CSIC Open Access Publication Support Initiative through its Unit of Information Resources for Research (URICI).

\section{Availability of data and materials}

The raw data and materials generated and/or analysed during the current study are available from the corresponding author on reasonable request.

Ethics approval and consent to participate Not applicable.
Consent for publication

Not applicable.

\section{Competing interests}

The authors declare that they have no competing interests.

\section{Author details}

${ }^{1}$ Present Address: R+D+I Department, Abiopep S.L., Murcia, Spain. ${ }^{2}$ Centro de Edafología y Biología Aplicada del Segura (CEBAS), Consejo Superior de Investigaciones Científicas (CSIC), Murcia, Spain.

Received: 25 February 2019 Accepted: 23 May 2019

Published online: 28 May 2019

\section{References}

1. Baltes NJ, Gil-Humanes J, Cermak T, Atkins PA, Voytas DF. DNA replicons for plant genome engineering. Plant Cell. 2014;26:151-63.

2. Mardanova ES, Blokhina EA, Tsybalova LM, Peyret H, Lomonossoff GP, Ravin NV. Efficient transient expression of recombinant proteins in plants by the novel pEff vector based on the genome of potato virus X. Front Plant Sci. 2017;8:1-8.

3. Yamamoto T, Hoshikawa K, Ezura K, Okazawa R, Fujita S, Takaoka M, et al. Improvement of the transient expression system for production of recombinant proteins in plants. Sci Rep. 2018;8:1-10.

4. Hefferon K. Plant virus expression vectors: a powerhouse for global health. Biomedicines. 2017:5:44.

5. Porta C, Lomonossoff GP. Viruses as vectors for the expression of foreign sequences in plants. Biotechnol Genet Eng Rev. 2002;19:245-92.

6. Liu L, Lomonossoff GP. Agroinfection as a rapid method for propagating Cowpea mosaic virus-based constructs. J Virol Methods. 2002;105:343-8.

7. Salazar-González JA, Bañuelos-Hernández B, Rosales-Mendoza S. Current status of viral expression systems in plants and perspectives for oral vaccines development. Plant Mol Biol. 2015;87:203-17.

8. Gleba Y, Klimyuk V, Marillonnet S. Viral vectors for the expression of proteins in plants. Curr Opin Biotechnol. 2007;18:134-41.

9. Lico C, Chen Q, Santi L. Viral vectors for production of recombinant proteins in plants. J Cell Physiol. 2008;216:366-77.

10. Peyret H, Lomonossoff GP. When plant virology met Agrobacterium: the rise of the deconstructed clones. Plant Biotechnol J. 2015;13:1121-35.

11. Sempere RN, Gómez P, Truniger $V$, Aranda MA. Development of expression vectors based on pepino mosaic virus. Plant Methods. 2011;7:6.

12. Aguilar JM, Hernández-Gallardo MD, Cenis JL, Lacasa A, Aranda MA. Complete sequence of the Pepino mosaic virus RNA genome. Arch Virol. 2002;147:2009-15.

13. Sempere RN, Gomez-Aix C, Ruiz-Ramon F, Gomez P, Hasiow-Jaroszewska B, Sanchez-Pina MA, et al. Pepino mosaic virus RNA-dependent RNA polymerase POL domain is a hypersensitive response-like elicitor shared by necrotic and mild isolates. Phytopathology. 2015;106:395-406.

14. Park M-R, Seo J-K, Kim K-H. Viral and nonviral elements in potexvirus replication and movement and in antiviral responses. Adv Virus Res. 2013:87:75-112.

15. Meng $M$, Lee CC. Function and structural organization of the replication protein of Bamboo mosaic virus. Front Microbiol. 2017;8:1-10.

16. Linnik O, Liesche J, Tilsner J, Oparka KJ. Unraveling the structure of viral replication complexes at super-resolution. Front Plant Sci. 2013:4:1-13.

17. Tilsner J, Linnik O, Wright KM, Bell K, Roberts AG, Lacomme C, et al. The TGB1 movement protein of potato virus $X$ reorganizes actin and endomembranes into the $\mathrm{X}$-body, a viral replication factory. Plant Physiol. 2012;158:1359-70.

18. Mathioudakis MM, Veiga RSL, Canto T, Medina V, Mossialos D, Makris AM, et al. Pepino mosaic virus triple gene block protein 1 (TGBp1) interacts with and increases tomato catalase 1 activity to enhance virus accumulation. Mol Plant Pathol. 2013;14:589-601.

19. Mathioudakis MM, Rodríguez-Moreno L, Sempere RN, Aranda MA, Livieratos I. Multifaceted capsid proteins: multiple interactions suggest multiple roles for Pepino mosaic virus capsid protein. Mol Plant-Microbe Interact. 2014;27:1356-69. 
20. Agirrezabala X, Méndez-López E, Lasso G, Sánchez-Pina MA, Aranda M, Valle $M$. The near-atomic cryoEM structure of a flexible filamentous plant virus shows homology of its coat protein with nucleoproteins of animal viruses. Elife. 2015;4:1-11.

21. Mathioudakis MM, Veiga R, Ghita M, Tsikou D, Medina V, Canto T, et al. Pepino mosaic virus capsid protein interacts with a tomato heat shock protein cognate 70. Virus Res. 2012;163:28-39.

22. Tilsner J, Linnik O, Louveaux M, Roberts IM, Chapman SN, Oparka KJ. Replication and trafficking of a plant virus are coupled at the entrances of plasmodesmata. J Cell Biol. 2013;201:981-95.

23. Lim HS, Vaira AM, Bae H, Bragg JN, Ruzin SE, Bauchan GR, et al. Mutation of a chloroplast-targeting signal in Alternanthera mosaic virus TGB3 impairs cell-to-cell movement and eliminates long-distance virus movement. J Gen Virol. 2010;91:2102-15.

24. Minicka J, Otulak K, Garbaczewska G, Pospieszny H, Hasiów-Jaroszewska B. Ultrastructural insights into tomato infections caused by three different pathotypes of Pepino mosaic virus and immunolocalization of viral coat proteins. Micron. 2015;79:84-92.

25. Lindbo JA. High-efficiency protein expression in plants from agroinfection-compatible Tobacco mosaic virus expression vectors. BMC Biotechnol. 2007;7:1-11.

26. Nelson BK, Cai X, Nebenführ A. A multicolored set of in vivo organelle markers for co-localization studies in Arabidopsis and other plants. Plant J. 2007:51:1126-36.

27. Cruz SS, Chapman S, Roberts AG, Roberts IM, Prior DA, Oparka KJ. Assembly and movement of a plant virus carrying a green fluorescent protein overcoat. Proc Natl Acad Sci. 1996:93:6286-90.

28. Gopinath K, Wellink J, Porta C, Taylor KM, Lomonossoff GP, Van Kammen A. Engineering cowpea mosaic virus RNA-2 into a vector to express heterologous proteins in plants. Virology. 2000;267:159-73.

29. Zhang C, Bradshaw JD, Whitham SA, Hill JH. The development of an efficient multipurpose bean pod mottle virus viral vector set for foreign gene expression and RNA silencing. Plant Physiol. 2010;153:52-65.

30. Tatineni S, McMechan AJ, Hein GL, French R. Efficient and stable expression of GFP through Wheat streak mosaic virus-based vectors in cereal hosts using a range of cleavage sites: Formation of dense fluorescent aggregates for sensitive virus tracking. Virology. 2011;410:268-81.

31. Minato N, Komatsu K, Okano Y, Maejima K, Ozeki J, Senshu H, et al. Efficient foreign gene expression in planta using a plantago asiatica mosaic virus-based vector achieved by the strong RNA-silencing suppressor activity of TGBp1. Arch Virol. 2014;159:885-96.

32. Röder J, Fischer R, Commandeur U. Adoption of the 2A ribosomal skip principle to tobacco mosaic virus for peptide display. Front Plant Sci. 2017:8:1-11.

33. Dawson WO, Lewandowski DJ, Hilf ME, Bubrick P, Raffo AJ, Shaw JJ, et al. A tobacco mosaic virus-hybrid expresses and loses an added gene. Virology. 1989;172:285-92.

34. Donson J, Kearney CM, Hilf ME, Dawson WO. Systemic expression of a bacterial gene by a tobacco mosaic virus-based vector. Proc Natl Acad Sci USA. 1991;88:7204-8.

35. Zhao Y, Hammond J, Tousignant ME, Hammond RW. Development and evaluation of a complementation-dependent gene delivery system based on cucumber mosaic virus. Arch Virol. 2000;145:2285-95.

36. Rabindran S, Dawson WO. Assessment of recombinants that arise from the use of a TMV-based transient expression vector. Virology 2001;284:182-9.
37. Haviv S, Galiakparov N, Goszczynski DE, Batuman O, Czosnek H, Mawassi $M$. Engineering the genome of Grapevine virus A into a vector for expression of proteins in herbaceous plants. JVirol Methods. 2006;132:227-31.

38. Draghici HK, Varrelmann M. Evidence for similarity-assisted recombination and predicted stem-loop structure determinant in potato virus $\mathrm{X}$ RNA recombination. J Gen Virol. 2010;91:552-62.

39. Nagy PD, Bujarski JJ. Efficient system of homologous RNA recombination in brome mosaic virus: sequence and structure requirements and accuracy of crossovers. J Virol. 1995;69:131-40.

40. Scholthof HB. Rapid delivery of foreign genes into plants by direct rubinoculation with intact plasmid DNA of a tomato bushy stunt virus gene vector. JVirol. 1999;73:7823-9.

41. Everett AL, Scholthof HB, Scholthof KBG. Satellite panicum mosaic virus coat protein enhances the performance of plant virus gene vectors. Virology. 2010;396:37-46.

42. Seaberg BL, Hsieh YC, Scholthof KBG, Scholthof HB. Host impact on the stability of a plant virus gene vector as measured by a new fluorescent local lesion passaging assay. J Virol Methods. 2012;179:289-94.

43. Asurmendi S, Berg RH, Koo JC, Beachy RN. Coat protein regulates formation of replication complexes during tobacco mosaic virus infection. Proc Natl Acad Sci. 2004;101:1415-20.

44. Miller S, Krijnse-Locker J. Modification of intracellular membrane structures for virus replication. Nat Rev Microbiol. 2008;6:363.

45. Den Boon JA, Diaz A, Ahlquist P. Cytoplasmic viral replication complexes. Cell Host Microbe. 2010:8:77-85.

46. Laliberté J-F, Sanfaçon H. Cellular remodeling during plant virus infection. Annu Rev Phytopathol. 2010;48:69-91.

47. Goldstein B. Cytological study of living cells of tobacco plants affected with mosaic disease. Bull Torrey Bot Club. 1924:51:261.

48. Dunoyer P, Ritzenthaler C, Hemmer O, Michler P, Fritsch C. Intracellular localization of the peanut clump virus replication complex in tobacco BY-2 protoplasts containing green fluorescent protein-labeled endoplasmic reticulum or golgi apparatus. JVirol. 2002;76:865-74.

49. Schwartz M, Chen J, Janda M, Sullivan M, Den Boon J, Ahlquist P. A positive-strand RNA virus replication complex parallels form and function of retrovirus capsids. Mol Cell. 2002;9:505-14.

50. Schwartz M, Chen J, Lee W-M, Janda M, Ahlquist P. Alternate, virusinduced membrane rearrangements support positive-strand RNA virus genome replication. Proc Natl Acad Sci. 2004;101:11263-8.

51. Sanfaçon H. Replication of positive-strand RNA viruses in plants: contact points between plant and virus components. Can J Bot. 2005;83:1529-49.

52. Le DHT, Méndez-López E, Wang C, Commandeur U, Aranda MA, Steinmetz NF. Biodistribution of filamentous plant virus nanoparticles: pepino mosaic virus versus potato virus X. Biomacromol. 2019;20:469-77.

53. Sambrook J, Russel D. Molecular cloning: a laboratory manual. 3rd ed. New York: Cold Spring Harbor Laboratory Press; 2001.

54. Nieto C, Morales M, Orjeda G, Clepet C, Monfort A, Sturbois B, et al. An elF4E allele confers resistance to an uncapped and non-polyadenylated RNA virus in melon. Plant J. 2006:48:452-62.

55. Sarrion-Perdigones A, Vazquez-Vilar M, Palaci J, Castelijns B, Forment J, Ziarsolo P, et al. GoldenBraid 2.0: a comprehensive DNA assembly framework for plant synthetic biology. Plant Physiol. 2013;162:1618-31.

\section{Publisher's Note}

Springer Nature remains neutral with regard to jurisdictional claims in published maps and institutional affiliations. 